\title{
Ruthenium-based Photosensitizer TLD1411
}

National Cancer Institute

\section{Source}

National Cancer Institute. Ruthenium-based Photosensitizer TLD1411. NCI Thesaurus.

Code $C 148182$

A ruthenium-based photosensitizer (PS), that can potentially be used for photodynamic therapy (PDT). Upon intratumoral administration, the ruthenium-based PS TLD1411 targets mitochondria in tumor cells. Upon PDT, TLD1411 produces singlet molecular oxygen (O2), and induces a reactive oxygen species (ROS)-mediated tumor cell apoptosis. 